\title{
Screening Methods for Isolation of Biocontrol Epiphytic Yeasts against Penicillium digitatum in Lemons
}

\author{
Martina María Pereyra ${ }^{1}$, Mariana Andrea Díaz ${ }^{1}$, Fabricio Fabián Soliz-Santander ${ }^{1} \mathbb{D}$, Anja Poehlein ${ }^{2} \mathbb{D}$, \\ Friedhelm Meinhardt ${ }^{3}\left(\mathbb{D}\right.$, Rolf Daniel ${ }^{2, * \mathbb{D}}$ and Julián Rafael Dib ${ }^{1,4, *}$
}

1 Planta Piloto de Procesos Industriales Microbiológicos (PROIMI)—Consejo Nacional de Investigaciones Científicas y Técnicas (CONICET), Av. Belgrano y Pje. Caseros, 4000 Tucumán, Argentina; mmpereyra@conicet.gov.ar (M.M.P.); marianadiaz@conicet.gov.ar (M.A.D.); fabianfabriciosoliz@gmail.com (F.F.S.-S.)

2 Genomic and Applied Microbiology \& Göttingen Genomics Laboratory, Institute of Microbiology and Genetics, Georg-August University of Göttingen, 37077 Göttingen, Germany; apoehle3@gwdg.de

3 Institut für Molekulare Mikrobiologie und Biotechnologie (IMMB), Westfälische Wilhelms Universität Münster, 48149 Münster, Germany; meinhar@uni-muenster.de

4 Facultad de Bioquímica, Instituto de Microbiología, Química y Farmacia, Universidad Nacional de Tucumán, Ayacucho 471, 4000 Tucumán, Argentina

* Correspondence: rdaniel@gwdg.de (R.D.); jdib@conicet.gov.ar (J.R.D.); Tel.: +49-(0)551-3933827 (R.D.); +54-(0)381-4344888 (J.R.D.)

check for updates

Citation: Pereyra, M.M.; Díaz, M.A.; Soliz-Santander, F.F.; Poehlein, A.; Meinhardt, F.; Daniel, R.; Dib, J.R. Screening Methods for Isolation of Biocontrol Epiphytic Yeasts against Penicillium digitatum in Lemons. J. Fungi 2021, 7, 166. https://doi.org/ 10.3390/jof7030166

Academic Editors: David S Perlin and Luis V. Lopez-Llorca

Received: 25 January 2021

Accepted: 22 February 2021

Published: 25 February 2021

Publisher's Note: MDPI stays neutral with regard to jurisdictional claims in published maps and institutional affiliations.

Copyright: (c) 2021 by the authors. Licensee MDPI, Basel, Switzerland. This article is an open access article distributed under the terms and conditions of the Creative Commons Attribution (CC BY) license (https:// creativecommons.org/licenses/by/ $4.0 /)$.

\begin{abstract}
Worldwide, the green rot caused by Penicillium digitatum is one of the most aggressive postharvest diseases of lemons. Searching for sustainable alternatives to chemical fungicides, epiphytic yeasts as potential biocontrol agents were isolated from citrus fruits using a tailor-made selective medium. For disclosing their antagonistic potential against $P$. digitatum, obtained isolates were subjected to direct screening methods, both in vitro and in vivo. In the course of the primary in vitro screening that comprised dual culture assays, 43 yeast strains displaying antagonistic activities against the pathogen were selected. Subsequently, such strains were subjected to an in vivo screening that consisted of a microscale test, allowing the selection of six yeast strains for further analysis. In the final screening using macroscale in vivo tests, three strains (AcL2, AgL21, and AgL2) displaying the highest efficiencies to control $P$. digitatum were identified. The protection efficiencies in lemons were 80 (AcL2), 76.7 (AgL21), and 75\% (AgL2). Based on sequence analysis of the PCR amplified D1/D2 domains of the 26S rRNA genes, they were identified as representatives of the species Clavispora lusitaniae. Interestingly, the strains exhibited a broad action spectrum among citrus fruits as they were also able to combat the green mold disease in grapefruit and two orange varieties. The direct screening methods applied in this study favored the recovery of efficient candidates for application as biological control agents to combat fungal infestations of citrus fruits.
\end{abstract}

Keywords: epiphytic yeasts; screening methods; biological control; Penicillium digitatum; citrus fruits

\section{Introduction}

Argentina is one of the main lemon-fruit-producing and exporting countries, with the Tucumán province being the lemon-producing hub contributing $78 \%$ of the total national production [1]. Lemons are frequently exposed to several phytosanitary issues that cause losses of up to $12 \%$ of the fresh fruit [1]; the green mold disease caused by Penicillium digitatum is the most important postharvest fungal infestation [2]. Traditionally, chemical control by the use of fungicides has been employed to control postharvest decays [3-5]. However, due to the emergence of fungicide-resistant pathogens in citrusproduction areas [3,6], the upcoming bans on the use of postharvest fungicides [7], and the public demand to reduce and avoid pesticides, are urgent needs to develop sustainable alternatives and safer technologies for controlling postharvest rots. Moreover, the mentioned 
drawbacks of fungicides have led markets to increase their demands to restrict chemical compounds, thereby promoting organic production of fruits and vegetables [8].

The use of naturally occurring epiphytic antagonists on fruit surfaces as biological control agents against postharvest pathogens is one of the most feasible alternatives to traditional fungicides. Selecting epiphytic antagonists from the environment where they are intended to be applied implies better adaptive advantages of the microorganisms $[9,10]$ and greater public acceptance [11]. Biocontrol agents such as bacteria, yeasts, fungi, and viruses can control plant diseases with direct or indirect antagonistic effects [12]. In particular, yeasts have attracted considerable interest due to their diverse mode of action, enabling them to combat fungal rots in fruit. The mode of action include wound colonization, competition for space and nutrients, inhibition of spore germination, secretion of extracellular enzymes [13-17], development of biofilms, production of siderophores or volatile compounds [18-20], and the killer phenotype [21] as direct mechanisms, as well as the induction of resistance in the respective plants [22] as an indirect mechanism. Indeed, the successful use of yeasts to control postharvest diseases has been reported for diverse crops such as apples [23,24], pears [25,26], grapes [27,28], strawberries [29,30], peaches [31], and citrus $[21,32,33]$.

A crucial step in the development of commercial products based on biological control agents is the screening and identification of suitable candidates. In our previous study, native yeasts for the postharvest control of P. digitatum were isolated from the surface of lemons and washing water from a local packinghouse [21]. As the latter sources showed to be adequate for isolation of efficient antagonistic yeasts, the current study aimed to increase the number of biocontrol candidates by using novel approaches for the isolation of naturally occurring epiphytic yeasts. The antagonistic activities of the biocontrol candidates were evaluated using different screening methods, in which the direct impact on the growth of the pathogen was measured. Furthermore, the biocontrol efficiency of the new isolates against green mold in lemons and other citrus fruits was evaluated.

\section{Materials and Methods}

\subsection{Fruits}

Lemons belonging to Eureka cultivars (Citrus limon (L.) Burm), sweet oranges variety Westin (Citrus sinensis), tangerine oranges (Citrus $x$ tangerine), and grapefruits (Citrus $x$ paradisis) were harvested from local fields in Tucumán province, Argentina. The selected cultivars had not received any preharvest treatment with synthetic pesticides. Healthy fruits were transported to the laboratory to be directly used or were stored at $8{ }^{\circ} \mathrm{C}$ for not more than 4 days. Selected fruits were free of any noticeable injury or signs of rot and were homogeneous in size, shape, and ripeness.

\subsection{Pathogen}

A phytopathogenic strain of $P$. digitatum belonging to the Phytopathology Lab of the citrus company San Miguel SA (Tucumán, Argentina) was used to generate green rot in lemons for the in vivo tests. The spore suspensions were prepared by collecting spores from a 10-day-old culture grown on PDA medium $\left(4 \mathrm{gL}^{-1}\right.$ potato extract, $20 \mathrm{gL}^{-1}$ glucose, $15 \mathrm{gL}^{-1}$ agar, $\mathrm{pH} 5.6$ ) at $25^{\circ} \mathrm{C}$. A total of $3 \mathrm{~mL}$ of saline solution containing $0.1 \%$ Tween 80 was added to the surface of the mycelium and scraped with a sterile loop. Spores were collected, and the suspension was adjusted to an $\mathrm{OD}_{600}$ of 0.1 , which corresponds to a concentration of $10^{6}$ spores $\mathrm{mL}^{-1}$ [21].

\subsection{Isolation of Epiphytic Yeasts}

Isolation of yeasts with potential antagonism against $P$. digitatum was carried out following two different strategies. In the first one, a non-selective method similar to that described by Chalutz and Wilson [34] was used. Sources of possible antagonistic agents were sampled from two different stages of fruit processing from a lemon packinghouse: $100 \mathrm{~mL}$ of water from the first fruit washing station (FWS) and $100 \mathrm{~mL}$ of essential oil from 
the first essential oil extraction station (EOES). Both types of samples were serially diluted, and $100 \mu \mathrm{L}$ of each dilution were subsequently plated in Petri dishes containing a modified YEPD-based medium called YEPD-CITRUS ( $5 \mathrm{gL}^{-1}$ yeast extract, $10 \mathrm{gL}^{-1}$ peptone, $20 \mathrm{gL}^{-1}$ glucose, $20 \mathrm{gL}^{-1}$ agar, $0.1 \%$ lemon essential oil, $1 \%$ lemon dehydrated peel powder, $\mathrm{pH} 4.5$ ) to simulate the natural environment of epiphytic yeasts. It was also supplemented with ampicillin $\left(100 \mu \mathrm{gmL}^{-1}\right)$ and chloramphenicol $\left(50 \mu \mathrm{gmL}^{-1}\right)$ to avoid bacterial contamination. After $24-72 \mathrm{~h}$ of incubation at $25^{\circ} \mathrm{C}$, individual colonies with different morphological appearances were purified, examined under bright field microscopy, and stored in $20 \%$ glycerol at $-80{ }^{\circ} \mathrm{C}$. The second isolation protocol was based on a selective method similar to the one described by Wilson et al. [35]. Twenty freshly-harvested lemons were first sanitized with a $70 \%$ ethanol solution and wounded on the equatorial side ( $3 \mathrm{~mm}$ deep and $2 \mathrm{~mm}$ wide) using an awl. Fruit wounds were directly inoculated with a $20 \mu \mathrm{L}$ sample (FWS or EOES). After $48 \mathrm{~h}$ incubation at $25^{\circ} \mathrm{C}, 20 \mu \mathrm{L}$ of the P. digitatum spore suspension was applied to each wound. Treated lemons were further incubated at $25^{\circ} \mathrm{C}$ with high relative humidity ( $95 \%)$ for 5 days. Wounds without evidence of green mold were scraped and washed with sterile saline solution $(0.85 \%)$. The obtained samples were serially diluted before spreading on YEPD-CITRUS plates and incubated at $25^{\circ} \mathrm{C}$ for $24-72 \mathrm{~h}$. Individual colonies were purified and stored as previously described.

\subsection{Selection of Antagonistic Yeasts}

\subsubsection{In Vitro Antagonistic Activity of Epiphytic Yeasts Against P. digitatum}

Isolated epiphytic yeasts were screened for their ability to inhibit fungal growth on PDA plates using a slightly modified dual culture assay [36]. A total of $5 \mu \mathrm{L}$ of the fungal spore suspension $\left(10^{6}\right.$ spores $\left.\mathrm{mL}^{-1}\right)$ was placed in the center of the Petri dishes $(90 \mathrm{~mm}$ diameter). On the other hand, a loop of tested yeast was streaked as a strip $20 \mathrm{~mm}$ from the edge and $25 \mathrm{~mm}$ from the central drop. The negative control consisted of PDA plates inoculated only with the fungal spore suspension. Plates were incubated at $25{ }^{\circ} \mathrm{C}$ for 10 days, and the relative degree of mycelial growth inhibition was calculated according to the diameter measurement data, comparing the growth diameter of the fungus in the dual culture with the growth of the fungus in the control. Experiments were conducted in triplicates.

\subsubsection{Biocontrol Assay on Lemon Fruit Against P. digitatum: Microscale and Macroscale Tests}

The efficiency of yeasts in wound protection against $P$. digitatum in lemons was first studied according to a microscale technique proposed by Ferraz et al. [37]. For this test, the best yeast candidates were selected based on the ability to inhibit $P$. digitatum in the in vitro tests. Yeast suspensions were prepared in $1 \mathrm{~mL}$ of saline solution using $24 \mathrm{~h}$ YEPD liquid cultures $\left(10^{8}\right.$ cells $\left.\mathrm{mL}^{-1}\right)$. Fifteen lemons per strain were surface disinfected, air-dried, and a single wound was introduced in the equatorial zone, as previously described. A total of $20 \mu \mathrm{L}$-aliquots of cell suspensions were inoculated into each wound, and treated fruits were incubated in a chamber under controlled conditions $\left(25^{\circ} \mathrm{C}\right.$ for $\left.24 \mathrm{~h}\right)$ before being inoculated with $20 \mu \mathrm{L}$ of the $P$. digitatum spore suspension. Fruits were stored in covered plastic containers for 5 days with $95 \%$ relative humidity. The infection control consisted of 15 lemons treated only with the pathogen. After the incubation period, the protection efficiency of tested yeasts was evaluated according to the number of healthy lemons per treatment using the following equation:

$$
\text { Protection efficiency }(\%)=\text { number of healthy fruit/total number of fruit }
$$

The best yeast candidates from the microscale screening method were employed for a further macroscale assay [21]. In this case, cell cultures were grown for $24 \mathrm{~h}$ in liquid YEPD, and aliquots were transferred to Erlenmeyer flasks containing $250 \mathrm{~mL}$ of the same medium. They were incubated for $48 \mathrm{~h}$ with shaking. Afterwards, yeast cells were recovered by centrifugation at $8000 \times \mathrm{rpm}$ for $5 \mathrm{~min}$ at $10^{\circ} \mathrm{C}$ (SLA-1500 rotor, Sorvall Instruments RCSC, Du Pont, Wilmington, DE, USA) and resuspended in standard saline solution reaching 
a final concentration of $10^{8}$ cells $\mathrm{mL}^{-1}$. A total of 60 lemons per yeast (4 replicates of 15 lemons) were used. Fruits were disinfected and wounded as described above and then placed in net bags to be immersed in the yeast suspensions. Yeast-treated lemons were incubated in a controlled chamber for $24 \mathrm{~h}$ at $25^{\circ} \mathrm{C}$ and, subsequently, immersed for $2 \mathrm{~min}$ in the fungus spore suspension. Yeast wound protection efficiency was evaluated after 5 days of incubation as detailed in the microscale test. A total of 20 lemons treated only with the pathogen served as the infection control in this assay.

Data were analyzed by ANOVA, and the mean values were compared with Tukey's test at the 5\% significance level. The InfoStat/L software (Córdoba, Argentina) [38] was used for the statistical analysis.

\subsection{Yeast DNA Extraction}

DNA extraction from isolated yeasts was performed following the methodology of Silverman [39] with slight modifications. Cell cultures were grown in $10 \mathrm{~mL}$ of YEPD medium at $25^{\circ} \mathrm{C}$ under shaking (170 rpm) for $48 \mathrm{~h}$, and $1 \mathrm{~mL}$ of each culture was pelleted by centrifugation at 13,000 $\times$ rpm (SLA1500 rotor, Sorvall Instruments RCSC, Du Pont) for $5 \mathrm{~min}$. Supernatants were discarded. The recovered pellets were incubated for $1 \mathrm{~h}$ at $37^{\circ} \mathrm{C}$ in a solution containing $500 \mu \mathrm{L}$ of Sorbitol $(1 \mathrm{M}), 100 \mu \mathrm{L}$ of EDTA buffer (pH 7.5), and $10 \mu \mathrm{L}$ of zymolyase $\left(2.5 \mathrm{mgmL}^{-1}\right.$, Zymo Research, Irvine, CA, USA). After incubation, samples were centrifuged under the above-mentioned conditions. The resulting pellets were suspended in $500 \mu \mathrm{L}$ of Tris-EDTA buffer ( $0.05 \mathrm{M}$ Tris, $0.02 \mathrm{M}$ EDTA, pH 7.4) and $50 \mu \mathrm{L}$ of $10 \%$ SDS before incubation at $65^{\circ} \mathrm{C}$ for $30 \mathrm{~min}$. Subsequently, $200 \mu \mathrm{L}$ of $5 \mathrm{M}$ potassium acetate was added, followed by incubation on ice for $1 \mathrm{~h}$ and centrifugation as described above. DNA precipitation from supernatants was carried out by adding one volume of isopropanol at room temperature $\left(5 \mathrm{~min}, 25^{\circ} \mathrm{C}\right)$, followed by centrifugation (10 $\mathrm{min}$, $13,000 \times$ rpm, SLA1500 rotor, Sorvall Instruments RCSC, Du Pont). Pellets were washed twice with $70 \%$ ethanol and allowed to dry. Finally, they were suspended in $100 \mu \mathrm{L}$ of TE buffer solution (10 mM Tris, $1 \mathrm{mM}$ EDTA, pH 7.4). DNA samples were analyzed using agarose $\left(0.8 \% \mathrm{wv}^{-1}\right)$ gel electrophoresis and stored at $-20{ }^{\circ} \mathrm{C}$ until further use.

Taxonomic identification was performed by PCR amplification of the D1/D2 domain of the $26 \mathrm{~S}$ rRNA gene using primers NL-1 (5'-GCA TAT CAA TAA GCG GAG GAA AAG-3') and NL-4 (5'-GGT CCG TGT TTC AAG ACG G-3') [40]. The PCR amplification mix (final volume, $50 \mu \mathrm{L}$ ) contained: $50-100 \mu \mathrm{g} \mu \mathrm{L}^{-1}$ of purified genomic DNA, $0.5 \mu \mathrm{M}$ of each primer, $200 \mu \mathrm{M}$ of deoxyribonucleoside triphosphate (dNTPs), $1 \times$ of Phusion High

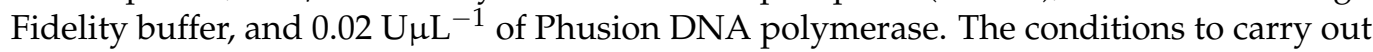
the amplification were the following: initial denaturation at $98^{\circ} \mathrm{C}$ for $30 \mathrm{~s}, 30$ cycles of $10 \mathrm{~s}$ at $98^{\circ} \mathrm{C}$, annealing at $63^{\circ} \mathrm{C}$ for $30 \mathrm{~s}$, extension at $72{ }^{\circ} \mathrm{C}$ for $15 \mathrm{~s}$, and the final extension at $72{ }^{\circ} \mathrm{C}$ for $5 \mathrm{~min}$. Amplified products were analyzed by $1 \%\left(\mathrm{wv}^{-1}\right)$ agarose gel electrophoresis. Sequencing of the purified PCR products was performed at Microsynth Seqlab (Göttingen, Germany). The obtained sequences were processed using Clone Manager 9 Software (Cary, NC, USA), and sequence similarity searches were performed with the BLAST network service of the NCBI database (http:/ / www.ncbi.nlm.nih.gov/BLAST). The sequences of these isolates have been deposited in the GenBank database under the following accession numbers: MT649495.1 (AcL2), MT649496.1 (AgL2), MT649498.1 (AgL21), MT649499.1 (AgRL4), MT649500.1 (AgRL5), and MT649497.1 (AgRL11).

\subsection{Yeasts Protection Efficiency Against P. digitatum in other Citrus Fruits}

The biocontrol spectrum of the selected yeasts against $P$. digitatum was evaluated using sweet oranges, tangerine oranges, and grapefruits. The experiment and the data analysis were carried out with the macroscale assay as described for lemons. 


\section{Results}

\subsection{Isolation and in Vitro Screening of Potential Biocontrol Epiphytic Yeasts}

Isolation of epiphytic yeasts was carried out using a novel modified YEPD medium added with lemon dehydrated peel powder and lemon essential oil (Figure 1). Two isolation strategies were adopted: a non-selective method in which the sources of antagonists were the FWS and the EOES; and a method whose selection was based on wounds of uninfected lemons, treated first with the foregoing samples and then with the pathogen. A total of 80 yeast strains were isolated: 56 from the non-selective method and 24 from the selective one (Table S1).

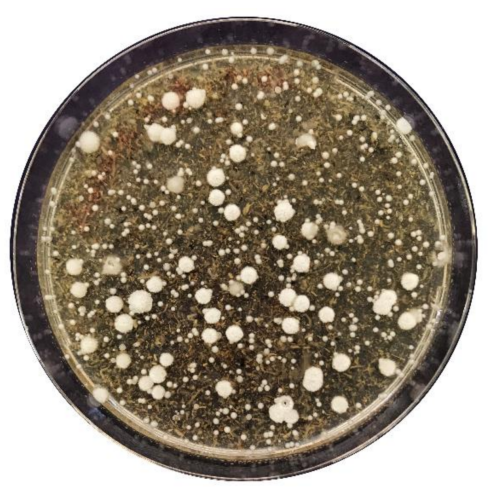

Figure 1. Yeast isolation on an agar plate using a modified YEPD medium. Yeast isolation from different antagonists' sources was carried out in a modified YEPD medium added with lemon dehydrated peel powder and lemon essential oil (YEPD-CITRUS). Selection was based on different morphological appearances, and they were examined under bright-field microscopy to confirm yeast morphology.

All isolated yeasts were primarily selected in an in vitro dual culture assay against P. digitatum. The antagonistic activity was determined by measuring the fungus relative growth inhibition after 10 days of incubation (Figure 2). Most of the strains (43) caused mycelial growth inhibition greater than 40\%, 22 caused inhibition between 15 and 40\%, 5 showed less than $15 \%$ inhibition, whereas the rest (10) did not affect the development of P. digitatum (Table S2).

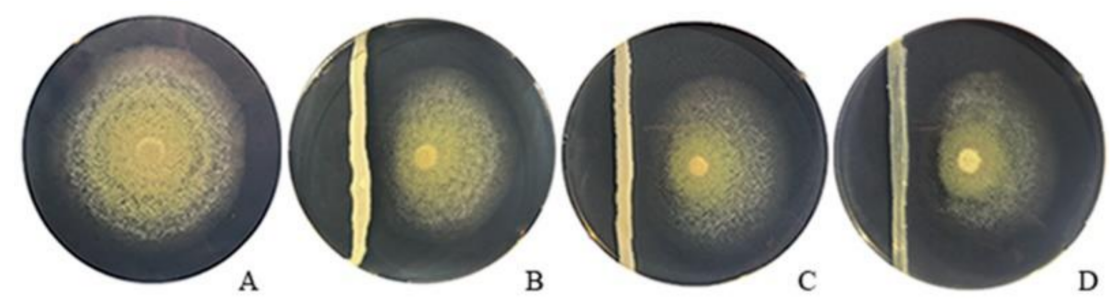

Figure 2. In vitro inhibitory activity of three isolated yeasts against $P$. digitatum on PDA medium after 10 days' incubation at $25^{\circ} \mathrm{C}$. (A) Control plate inoculated only with the pathogen. (B-D) Plates inoculated with the pathogen and a strip of yeasts: AgL2, AgL21, and AcL2, respectively.

\subsection{In Vivo Screening Methods against P. digitatum in Lemons}

In vivo tests were conducted with the preselected isolates from the in vitro assay. The best 43 candidates were first evaluated in a microscale test against the pathogen. After 5 days of incubation at $25^{\circ} \mathrm{C}$, most of the yeasts were able to control green mold in wounded fruits (Figure 3). Wound protection efficiencies of at least $80 \%$ were adopted as the selection criterion for antagonistic yeasts to be further evaluated in the macroscale test, confirming their biocontrol activity against $P$. digitatum. Isolates that complied with this microscale test requirement were AgL2, AgL21, AcL2, AgRL4, AgRL5, and AgRL11. AcL2, 
AgL21, and AgL2 showed to be the most protective candidates in the in vivo macroscale test with efficiencies of 80,76.7, and 75\%, respectively (Figure 4).

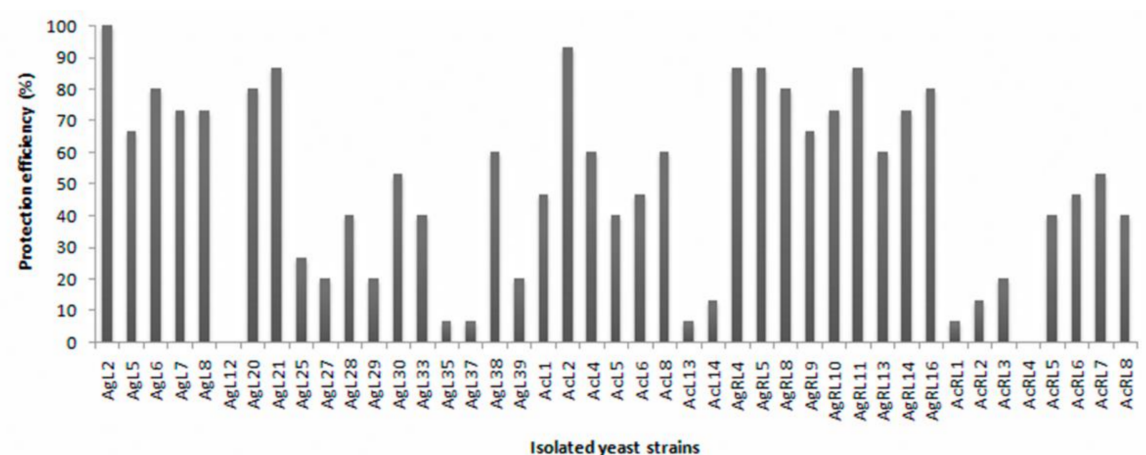

Figure 3. Wound protection efficiency of yeasts in the in vivo microscale assay. The 43 yeasts that most inhibited the mycelial growth of $P$. digitatum in the in vitro test were evaluated in a microscale assay against the pathogen after 5 days of incubation at $25^{\circ} \mathrm{C}$.
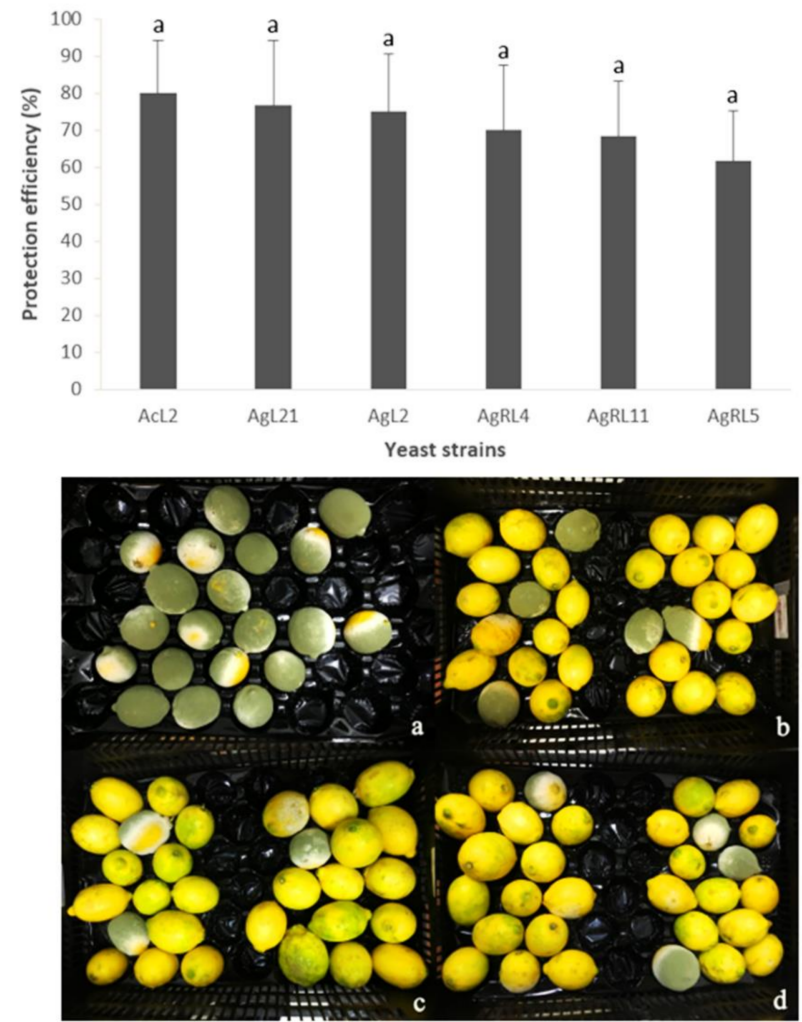

A

Figure 4. Wound protection efficiencies and biocontrol test of selected candidates. The upper panel (A) shows yeast wound protection efficiencies in the in vivo macroscale assay. The best yeast candidates obtained from the microscale assay were evaluated against $P$. digitatum after 5 days of incubation at $25{ }^{\circ} \mathrm{C}$. Error bars indicate standard deviations. The bottom panel (B) represents the in vivo macroscale biocontrol test in lemons. The figure shows wound protection in lemons inoculated only with P. digitatum (a), compared to lemons pretreated with yeasts (b) AgL21, (c) AgL2, and (d) AcL2 after 5 days at $25^{\circ} \mathrm{C}$.

\subsection{Identification of Antagonistic Yeasts}

According to the sequence analysis of the D1/D2 domain of the 26S rRNA gene and the search for similarities in the GenBank database, isolated yeasts were all affiliated to the 
Saccharomycetaceae family of the order Saccharomycetales. AgL2, AgL21, AcL2, AgRL4, AgRL5, and AgRL11 were identified as Clavispora lusitaniae, showing an identity greater than $99.5 \%$ to the reference strain C. lusitaniae Y8 (MN648842.1) (Table 1).

Table 1. Identification of the yeast species of the six best isolates.

\begin{tabular}{ccccc}
\hline Isolate & Fragment Length $^{\mathbf{a}}$ & $\begin{array}{c}\text { Species } \\
\text { Designation }\end{array}$ & $\begin{array}{c}\text { GenBank } \\
\text { Accession Number }\end{array}$ & Identity (\%) $^{\mathbf{b}}$ \\
\hline AcL2 & 461 & Clavispora lusitaniae & MT649495.1 & 100 \\
AgL2 & 461 & Clavispora lusitaniae & MT649496.1 & 100 \\
AgL21 & 461 & Clavispora lusitaniae & MT649498.1 & 100 \\
AgRL4 & 459 & Clavispora lusitaniae & MT649499.1 & 99.57 \\
AgRL5 & 461 & Clavispora lusitaniae & MT649500.1 & 99.57 \\
AgRL11 & 459 & Clavispora lusitaniae & MT649497.1 & 99.57 \\
\hline
\end{tabular}

${ }^{a}$ Values refer to the number of base pairs per fragment; ${ }^{b}$ Identical nucleotides percentage in the sequence obtained from the D1/D2 region of the 26S rDNA gene and the sequence found in GenBank.

\subsection{Biocontrol Efficiency against P. digitatum in other Citrus Fruits}

The three candidates selected for the in vivo macroscale assay were evaluated with respect to protection activity against green mold in other citrus fruits to assess their biocontrol spectrum. Yeasts showed efficiency in controlling green mold in sweet oranges, tangerine oranges, and grapefruits (Figure 5). AcL2 and AgL21 showed the highest protection efficiency for tangerine oranges and grapefruits (95 and 97.5\%, respectively), whereas sweet oranges protection was significantly lower in both cases (AcL2 57.5\% and AgL21 72.5\%). Strain AgL2 was able to control the green mold in all tested citrus, with protection efficiencies of $77.5,92.5$, and $92.5 \%$ for sweet oranges, tangerine oranges, and grapefruits, respectively. It should be noted that there were no significant differences in protection when comparing the protective effects of AcL2, AgL2, and AgL21 against the same type of citrus fruit.
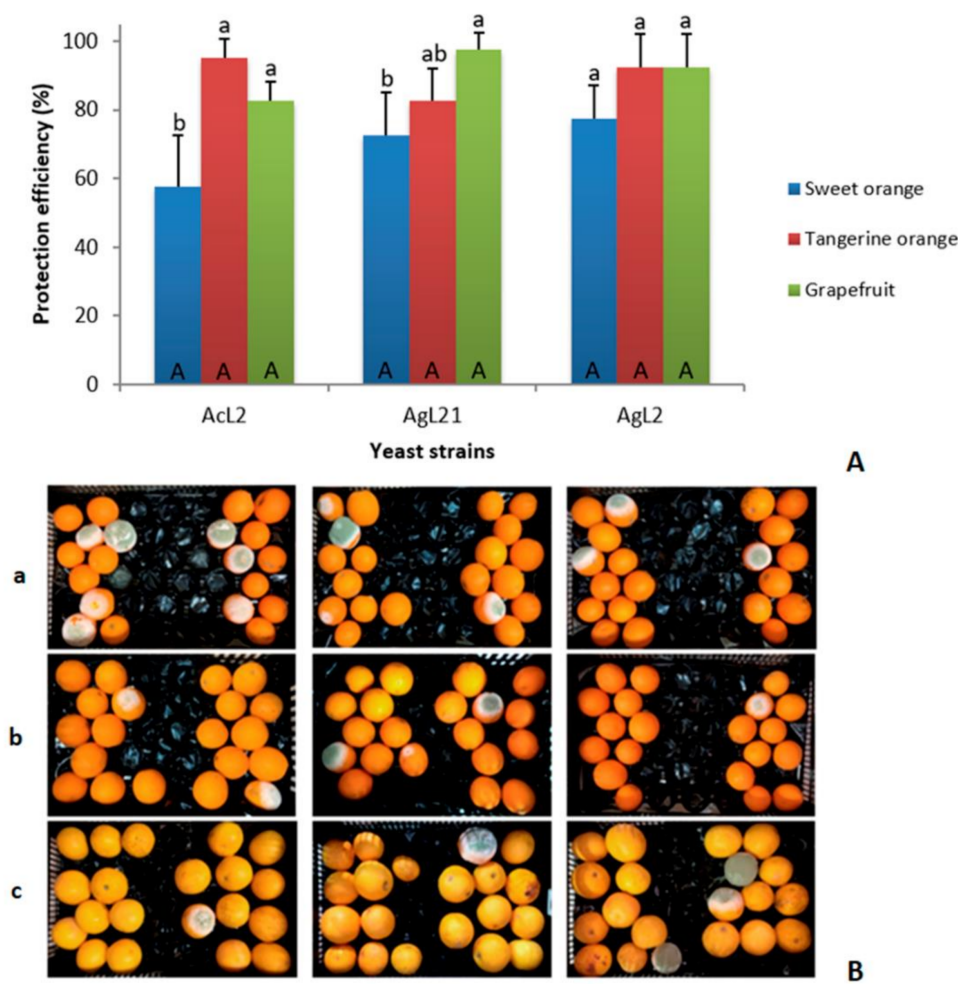

Figure 5. Protection efficiencies of potential yeast candidates against $P$. digitatum in citrus fruits. The upper panel (A) shows the protection efficiency of the three isolates against the pathogen in citrus cultivars. Mean values marked with identical letters are, according to the Tukey test $(p<0.05)$, not 
significantly different. Lowercase letters compare the efficiency of each yeast in the three citrus varieties. Uppercase letters represent the efficiencies comparison among the three yeasts in the same variety. The bottom panel (B) represents the in vivo test after 5 days of incubation at $25^{\circ} \mathrm{C}$. The efficiency of the yeasts AcL2, AgL21, and AgL2 (sorted by column, respectively) was evaluated in sweet oranges (a), tangerine oranges (b), and grapefruits (c).

\section{Discussion}

The drawbacks associated with the use of synthetic fungicides in the control of postharvest fungal diseases in lemons, followed by the growing demand for organic products, have encouraged the search and development of effective and more sustainable alternatives for the control of postharvest decays. In this regard, biological control agents based on yeasts have shown great potential as an alternative to the use of fungicides.

The main objective of this study was to isolate and select potential biocontrol yeasts to prevent or reduce infection by $P$. digitatum by using different direct screening methods. Most studies report the isolation of biological control agents from the region in which the final application is intended. This strategy is recommended to obtain microorganisms adapted to the environment, ensure their survival, and enhance their biocontrol activity in terms of their possible commercialization [11,41]. The isolation strategies described in this study allowed the selection of efficient antagonistic yeasts for the control of postharvest diseases of fruits. On the one hand, the presence of candidate yeasts was determined by a non-selective method in which the source of antagonistic agents was directly the FWS and the EOES. On the other hand, a selective isolation method was carried out from the same samples. Our results showed that the highest number of isolates was obtained from the non-selective method (Table S1). Of the yeasts isolated by the selective method, $70.8 \%$ had antagonistic activity against $P$. digitatum, whereas only $46.4 \%$ of those obtained by the non-selective approach showed such activity (Table S2). These results are in line with those of Wilson et al. [35] and Huang et al. [42], who argued that the selective isolation method is highly effective and should be considered as a first option for the sampling of biocontrol agents. Similarly, Taqarort et al. [43] obtained a high number of antagonistic yeasts using a selective method. Additionally, a modified medium was used to promote yeast development by adding lemon peel powder and lemon essential oil. This enrichment strategy proved to be an efficient approach to isolate yeasts that could serve as potential biocontrol agents against postharvest diseases of lemons. Similarly, Vero et al. [23] isolated yeasts capable of colonizing apple wounds and prevent the development of Penicillium expansum and Botrytis cinerea by adding apple juice to the culture medium.

Regarding the initial screening method used, the primary selection of biological control agents through in vitro dual culture assays has proven to be a simple, fast, and reproducible way to identify microorganisms with confirmed in vivo biocontrol activity $[21,23,43]$. In this study, 43 isolated yeasts were able to inhibit more than $40 \%$ of the mycelial growth of $P$. digitatum in the in vitro screening. However, it is important to mention that the screening of biocontrol agents by a dual culture assay is obviously restricted to those with direct activity against the pathogen, limiting the possibility of finding other promising biocontrol agents [41].

In a second selection screening consisting of a microscale in vivo test, in which both yeast and fungus were applied directly onto the lemon wound, the number of possible yeast candidates against $P$. digitatum was considerably reduced. The best yeast strains were selected according to their highest wound protection efficiencies in the microscale test: AgL2 100\%, AcL2 93.33\%, AgL21, AgRL4, AgRL5, and AgRL11 86.67\%. Several studies reported the finding of successful antagonistic yeasts by employing such a methodology for controlling B. cinerea in apples [15] and grapes [44], P. digitatum in citrus [45-47], and P. expansum in pears [25]. Yeast strains AgL2, AcL2, AgL21, AgRL4, AgRL5, and AgRL11 not only inhibited the mycelial growth of $P$. digitatum in vitro but also prevented the pathogen development in fruits. Nevertheless, it should be noted that when the same 
yeasts were tested in the macroscale in vivo assay, their biocontrol efficiencies were lower, reaching values ranging from 61 to $80 \%$. This highlights the importance of both choosing a suitable screening method for selecting yeasts and correctly selecting how to apply the biocontrol agent. For example, inoculation of the microbial agent directly into the fruit wound by the microscale method is a widely used technique due to its speed and easiness, but it certainly does not represent conventional application methods used under fruit packaging conditions.

In this study, the most promising yeast isolates (AgL2, AcL21, AgL21, AgRL4, AgRL5, and AgRL11) were identified by sequencing the D1/D2 region of the 26S rRNA gene and were all identified as representatives of Clavispora lusitaniae. This is consistent with our previous reports [21] in which C. lusitaniae strain 146 acted as an efficient biological control agent against $P$. digitatum, including fungicide-resistant $P$. digitatum strains [48]. In the present study, new methodologies have been tested to favor the isolation and selection of biocontrol agents: (i) the isolation of yeasts from two different samples (the FWS and the EOES), (ii) the use of a modified culture medium to simulate the natural environment of lemon epiphytic yeasts, favoring their development, (iii) the use of a selection method to obtain yeasts from uninfected lemon wounds previously inoculated with the industrial samples described here, and (iv) the implementation of different direct screening methods for an appropriate selection of candidate yeasts as biocontrol agents. Hence, these novel procedures led us to isolate members of the most efficient genus according to our own previous studies $[48,49]$. Apparently, members of the species C. lusitaniae seem to be native citrus yeasts with strong protective impacts on green rot in lemons. Furthermore, our group demonstrated recently that $C$. lusitaniae 146 is highly tolerant to certain stress factors associated with lemon storage and packaging processes, such as oxidative stress, fruit drying temperature, salts, and disinfectants commonly used in the citrus industry as well as UV-B irradiation [50]. Resistance to various stressors could explain the abundance of these yeast species in the isolation sources.

C. lusitaniae strains AgL2, AgL21, and AcL2 behaved as broad control agents among citrus fruits, as other than lemons, they controlled the green mold in oranges and grapefruits as well. The selection of a biocontrol agent with a broad spectrum of activity is a commercially highly desirable trait [51], which increases the application possibilities of formulations based on such yeasts. It is, thus, not surprising that other biocontrol yeasts such as Aureobasidium pullulans [52] and Candida oleophila [53,54], available in commercial formulations [17], also exhibit a wide spectrum of action in different crops.

\section{Conclusions}

By employing novel isolation and screening approaches, it was possible to obtain native epiphytic yeasts with effective antagonistic activity from citrus sources. It is noteworthy that any selection method is driven by certain interests and, hence, will be selective, which implies that not all candidate microorganisms suitable to act as biocontrol agents will be detected. The C. lusitaniae isolates AcL2, AgL2, and AgL21 were the most efficient in controlling the most important postharvest pathogen of lemons, both in vitro and in vivo. In addition, they proved to be agents with a broad activity spectrum, managing to control green mold in different varieties of citrus. Thus, the selected strains expand the collection of candidate yeasts for possible applications as alternative biological control agents against postharvest fungal diseases. Currently, in addition to evaluating the safety of the biocontrol yeasts with respect to human health, the mechanisms of action by which these new isolates exert their biocontrol activity are being studied, which certainly will also contribute to the understanding of the protective effects of yeasts against other postharvest citrus phytopathogens.

Supplementary Materials: The following is available online at https:/ / www.mdpi.com/2309-608 X/7/3/166/s1, Table S1: Number of isolated yeasts according to source and employed method; Table S2: Relative growth inhibition of yeast strains against $P$. digitatum. 
Author Contributions: Conceptualization, J.R.D.; Formal analysis, M.M.P., M.A.D., R.D., and J.R.D.; Funding acquisition, R.D. and J.R.D.; Investigation, M.M.P., M.A.D., and A.P.; Methodology, M.M.P., M.A.D., F.F.S.-S., and A.P.; Project administration, R.D. and J.R.D.; Supervision, R.D. and J.R.D.; Visualization, M.M.P.; Writing-original draft, M.M.P., F.M., R.D., and J.R.D.; Writing-review \& editing, M.M.P., F.M., R.D., and J.R.D. All authors have read and agreed to the published version of the manuscript.

Funding: This work was partially funded by Proyecto PIUNT A618/2 and PICT 2018-2545 PRESTAMO BID. Julián Rafael Dib thanks the support of the Alexander von Humboldt Foundation.

Institutional Review Board Statement: Not applicable.

Informed Consent Statement: Not applicable.

Data Availability Statement: The data presented in this study are available in insert article or supplementary material here.

Acknowledgments: We thank Ing. Gustavo Dib and Ing. Gabriel Ross for the provision of fresh fruit.

Conflicts of Interest: The authors declare no conflict of interest. The funders had no role in the design, execution, interpretation, or writing of the study.

\section{References}

1. Federcitrus la Actividad Citrícola Argentina. Available online: https://www.federcitrus.org/estadisticas/ (accessed on 28 September 2020).

2. Bancroft, M.; Gardner, P.; Eckert, J.; Baritelle, J. Comparison of decay control strategies in California lemon packinghouses. Plant Dis. 1984, 68, 24. [CrossRef]

3. Eckert, J.W.; Sievert, J.R.; Ratnayake, M. Reduction of imazalil effectiveness against citrus green mold in California packinghouses by resistant biotypes of Penicillium digitatum. Plant Dis. 1994, 78, 971-974. [CrossRef]

4. Kanetis, L.; Förster, H.; Adaskaveg, J. Optimizing efficacy of new postharvest fungicides and evaluation of sanitizing agents for managing citrus green mold. Plant Dis. 2008, 92, 261-269. [CrossRef] [PubMed]

5. Sepulveda, M.; Cuevas, I.I.; Smilanick, J.L.; Cerioni, L.; Rapisarda, V.A.; Ramallo, J. Improvement in imazalil treatments in commercial packinglines to control green mold on lemon fruit. Sci. Hortic. 2015, 192, 387-390. [CrossRef]

6. Fogliata, G.; Torres Leal, G.; Ploper, L. Detection of imazalil-resistant strains of Penicillium digitatum Sacc. in citrus packinghouses of Tucumán Province (Argentina) and their behavior against currently employed and alternative fungicides. Revista Industrial Agrícola Tucumán 2000, 77, 71-75.

7. Brancato, A.; Brocca, D.; Carrasco Cabrera, L.; de Lentdecker, C.; Erdos, Z.; Ferreira, L.; Greco, L.; Jarrah, S.; Kardassi, D.; Leuschner, R.; et al. Updated review of the existing maximum residue levels for imazalil according to Article 12 of Regulation (EC) No 396/2005 following new toxicological information. EFSA J. 2018, 16. [CrossRef]

8. Teixidó, N.; Vinas, I.; Usall, J.; Sanchis, V.; Magan, N. Ecophysiological responses of the biocontrol yeast Candida sake to water, temperature and pH stress. J. Appl. Microbiol. 1998, 84, 192-200. [CrossRef]

9. Bull, C.T.; Stack, J.P.; Smilanick, J.L. Pseudomonas syringae strains ESC-10 and ESC-11 survive in wounds on citrus and control green and blue molds of citrus. Biol. Control 1997, 8, 81-88. [CrossRef]

10. Pimenta, R.S.; Morais, P.B.; Rosa, C.A.; Corrêa, A. Utilization of Yeasts in Biological Control Programs. In Yeast Biotechnology: Diversity and Applications; Springer: Berlin/Heidelberg, Germany, 2009; pp. 199-214.

11. Wilson, C.L.; Wisniewski, M.E. Biological control of postharvest diseases of fruits and vegetables: An emerging technology. Annu. Rev. Phytopathol. 1989, 27, 425-441. [CrossRef]

12. Köhl, J.; Kolnaar, R.; Ravensberg, W.J. Mode of action of microbial biological control agents against plant diseases: Relevance beyond efficacy. Front. Plant Sci. 2019, 10. [CrossRef] [PubMed]

13. Grevesse, C.; Lepoivre, P.; Jijakli, M.H. Characterization of the exoglucanase-encoding gene PaEXG2 and study of its role in the biocontrol activity of Pichia anomala strain K. Phytopathology 2003, 93, 1145-1152. [CrossRef]

14. Wisniewski, M.; Wilson, C.; Droby, S.; Chalutz, E.; El-Ghaouth, A.; Stevens, C. Postharvest Biocontrol: New Concepts and Applications. In Biological Control: A Global Perspective; Cabi Publishing: Wallingford, UK, 2007; pp. $262-273$.

15. Vero, S.; Garmendia, G.; González, M.B.; Garat, M.F.; Wisniewski, M. Aureobasidium pullulans as a biocontrol agent of postharvest pathogens of apples in Uruguay. Biocontrol Sci. Technol. 2009, 19, 1033-1049. [CrossRef]

16. Lutz, M.C.; Lopes, C.A.; Rodriguez, M.E.; Sosa, M.C.; Sangorrín, M.P. Efficacy and putative mode of action of native and commercial antagonistic yeasts against postharvest pathogens of pear. Int. J. Food Microbiol. 2013, 164, 166-172. [CrossRef]

17. Spadaro, D.; Droby, S. Development of biocontrol products for postharvest diseases of fruit: The importance of elucidating the mechanisms of action of yeast antagonists. Trends Food Sci. Technol. 2016, 47, 39-49. [CrossRef]

18. Giobbe, S.; Marceddu, S.; Scherm, B.; Zara, G.; Mazzarello, V.L.; Budroni, M.; Migheli, Q. The strange case of a biofilm-forming strain of Pichia fermentans, which controls Monilinia brown rot on apple but is pathogenic on peach fruit. FEMS Yeast Res. 2007, 7 , 1389-1398. [CrossRef] 
19. Wang, W.L.; Chi, Z.M.; Chi, Z.; Li, J.; Wang, X.H. Siderophore production by the marine-derived Aureobasidium pullulans and its antimicrobial activity. Bioresour. Technol. 2009, 100, 2639-2641. [CrossRef] [PubMed]

20. Arrarte, E.; Garmendia, G.; Rossini, C.; Wisniewski, M.; Vero, S. Volatile organic compounds produced by Antarctic strains of Candida sake play a role in the control of postharvest pathogens of apples. Biol. Control 2017, 109, 14-20. [CrossRef]

21. Perez, M.F.; Contreras, L.; Garnica, N.M.; Fernández-Zenoff, M.V.; Farías, M.E.; Sepulveda, M.; Ramallo, J.; Dib, J.R. Native killer yeasts as biocontrol agents of postharvest fungal diseases in lemons. PLoS ONE 2016, 11. [CrossRef] [PubMed]

22. Yao, H.J.; Tian, S.P. Effects of a biocontrol agent and methyl jasmonate on postharvest diseases of peach fruit and the possible mechanisms involved. J. Appl. Microbiol. 2005, 98, 941-950. [CrossRef] [PubMed]

23. Vero, S.; Garmendia, G.; González, M.B.; Bentancur, O.; Wisniewski, M. Evaluation of yeasts obtained from Antarctic soil samples as biocontrol agents for the management of postharvest diseases of apple (Malus $\times$ domestica). FEMS Yeast Res. 2013, 13, 189-199. [CrossRef]

24. Czarnecka, M.; Żarowska, B.; Połomska, X.; Restuccia, C.; Cirvilleri, G. Role of biocontrol yeasts Debaryomyces hansenii and Wickerhamomyces anomalus in plants' defence mechanisms against Monilinia fructicola in apple fruits. Food Microbiol. 2019, 83, 1-8. [CrossRef]

25. Zhang, Q.; Zhao, L.; Li, Z.; Li, C.; Li, B.; Gu, X.; Zhang, X.; Zhang, H. Screening and identification of an antagonistic yeast controlling postharvest blue mold decay of pears and the possible mechanisms involved. Biol. Control 2019, 133, 26-33. [CrossRef]

26. Lutz, M.C.; Lopes, C.A.; Sosa, M.C.; Sangorrín, M.P. Semi-commercial testing of regional yeasts selected from North Patagonia Argentina for the biocontrol of pear postharvest decays. Biol. Control 2020, 150. [CrossRef]

27. Senthil, R.; Prabakar, K.; Rajendran, L.; Karthikeyan, G. Efficacy of different biological control agents against major postharvest pathogens of grapes under room temperature storage conditions. Phytopathol. Mediterr. 2011, 50, 55-64. [CrossRef]

28. Ponsone, M.L.; Nally, M.C.; Chiotta, M.L.; Combina, M.; Köhl, J.; Chulze, S.N. Evaluation of the effectiveness of potential biocontrol yeasts against black sur rot and ochratoxin a occurring under greenhouse and field grape production conditions. Biol. Control 2016, 103, 78-85. [CrossRef]

29. Zhang, H.; Zheng, X.; Wang, L.; Li, S.; Liu, R. Effect of yeast antagonist in combination with hot water dips on postharvest Rhizopus rot of strawberries. J. Food Eng. 2007, 78, 281-287. [CrossRef]

30. Huang, R.; Che, H.J.; Zhang, J.; Yang, L.; Jiang, D.H.; Li, G.Q. Evaluation of Sporidiobolus pararoseus strain YCXT3 as biocontrol agent of Botrytis cinerea on post-harvest strawberry fruits. Biol. Control 2012, 62, 53-63. [CrossRef]

31. Zhang, D.; Spadaro, D.; Garibaldi, A.; Gullino, M.L. Selection and evaluation of new antagonists for their efficacy against postharvest brown rot of peaches. Postharvest Biol. Technol. 2010, 55, 174-181. [CrossRef]

32. Platania, C.; Restuccia, C.; Muccilli, S.; Cirvilleri, G. Efficacy of killer yeasts in the biological control of Penicillium digitatum on Tarocco orange fruits (Citrus sinensis). Food Microbiol. 2012, 30, 219-225. [CrossRef] [PubMed]

33. Liu, J.; Sui, Y.; Wisniewski, M.; Droby, S.; Liu, Y. Review: Utilization of antagonistic yeasts to manage postharvest fungal diseases of fruit. Int. J. Food Microbiol. 2013, 167, 153-160. [CrossRef]

34. Chalutz, E.; Wilson, C. Postharvest biocontrol of green and blue mold and sour rot of citrus fruit by Debaryomyces hansenii. Plant Dis. 1990, 74, 134. [CrossRef]

35. Wilson, C.L.; Wisniewski, M.E.; Droby, S.; Chalutz, E. A selection strategy for microbial antagonists to control postharvest diseases of fruits and vegetables. Sci. Hortic. 1993, 53, 183-189. [CrossRef]

36. Spadaro, D.; Vola, R.; Piano, S.; Gullino, M.L. Mechanisms of action and efficacy of four isolates of the yeast Metschnikowia pulcherrima active against postharvest pathogens on apples. Postharvest Biol. Technol. 2002, 24, 123-134. [CrossRef]

37. Ferraz, L.P.; da Cunha, T.; da Silva, A.C.; Kupper, K.C. Biocontrol ability and putative mode of action of yeasts against Geotrichum citri-aurantii in citrus fruit. Microbiol. Res. 2016, 188-189, 72-79. [CrossRef] [PubMed]

38. Di-Rienzo, J.; Casanoves, F.; Balzarini, M.; Gonzalez, L. InfoStat Versión Grupo InfoStat; Universidad Nacional de Córdoba: Córdoba, Argentina, 2009.

39. Silverman, S. Methods in yeast genetics (laboratory course manual). Anal. Biochem. 1987, 167, 424. [CrossRef]

40. Sugita, T.; Takashima, M.; Kodama, M.; Tsuboi, R.; Nishikawa, A. Description of a new yeast species, Malassezia japonica, and its detection in patients with atopic dermatitis and healthy subjects. J. Clin. Microbiol. 2003, 41, 4695-4699. [CrossRef] [PubMed]

41. Raymaekers, K.; Ponet, L.; Holtappels, D.; Berckmans, B.; Cammue, B.P.A. Screening for novel biocontrol agents applicable in plant disease management-A review. Biol. Control 2020, 104240. [CrossRef]

42. Huang, J.; Wei, Z.; Tan, S.; Mei, X.; Yin, S.; Shen, Q.; Xu, Y. The rhizosphere soil of diseased tomato plants as a source for novel microorganisms to control bacterial wilt. Appl. Soil Ecol. 2013, 72, 79-84. [CrossRef]

43. Taqarort, N.; Echairi, A.; Chaussod, R.; Nouaim, R.; Boubaker, H.; Benaoumar, A.A.; Boudyach, E. Screening and identification of epiphytic yeasts with potential for biological control of green mold of citrus fruits. World J. Microbiol. Biotechnol. 2008, 24, 3031-3038. [CrossRef]

44. Parafati, L.; Vitale, A.; Restuccia, C.; Cirvilleri, G. Biocontrol ability and action mechanism of food-isolated yeast strains against Botrytis cinerea causing post-harvest bunch rot of table grape. Food Microbiol. 2015, 47, 85-92. [CrossRef]

45. Mekbib, S.B.; Regnier, T.J.C.; Korsten, L. Efficacy and mode of action of yeast antagonists for control of Penicillium digitatum in oranges. Trop. Plant Pathol. 2011, 36, 233-240. [CrossRef]

46. Sperandio, E.M.; Martins do Vale, H.M.; Moreira, G.A.M. Yeasts from native Brazilian Cerrado plants: Occurrence, diversity and use in the biocontrol of citrus green mould. Fungal Biol. 2015, 119, 984-993. [CrossRef] 
47. Liu, Y.; Wang, W.; Zhou, Y.; Yao, S.; Deng, L.; Zeng, K. Isolation, identification and In Vitro screening of Chongqing orangery yeasts for the biocontrol of Penicillium digitatum on citrus fruit. Biol. Control 2017, 110, 18-24. [CrossRef]

48. Perez, M.F.; Díaz, M.A.; Pereyra, M.M.; Córdoba, J.M.; Isas, A.S.; Sepúlveda, M.; Ramallo, J.; Dib, J.R. Biocontrol features of Clavispora lusitaniae against Penicillium digitatum on lemons. Postharvest Biol. Technol. 2019, 155, 57-64. [CrossRef]

49. Perez, M.F.; Perez Ibarreche, J.; Isas, A.S.; Sepulveda, M.; Ramallo, J.; Dib, J.R. Antagonistic yeasts for the biological control of Penicillium digitatum on lemons stored under export conditions. Biol. Control 2017, 115, 135-140. [CrossRef]

50. Pereyra, M.M.; Díaz, M.A.; Meinhardt, F.; Dib, J.R. Effect of stress factors associated with postharvest citrus conditions on the viability and biocontrol activity of Clavispora lusitaniae strain. PLoS ONE 2020, 15, e0239432. [CrossRef] [PubMed]

51. Corrêa, B.O.; Schafer, J.T.; Moura, A.B. Spectrum of biocontrol bacteria to control leaf, root and vascular diseases of dry bean. Biol. Control 2014, 72, 71-75. [CrossRef]

52. Chi, Z.; Wang, F.; Chi, Z.; Yue, L.; Liu, G.; Zhang, T. Bioproducts from Aureobasidium pullulans, a biotechnologically important yeast. Appl. Microbiol. Biotechnol. 2009, 82, 793-804. [CrossRef]

53. Ballet, N.; Souche, J.L.; Vandekerckove, P. Efficacy of Candida Oleophila, Strain O, in Preventing Postharvest Diseases of Fruits. In Proceedings of the III International Symposium on Postharvest Pathology: Using Science to Increase Food Availability, Bari, Italy, 7 November 2016; Volume 1144, pp. 105-111.

54. Sui, Y.; Sun, Z.; Zou, Y.; Li, W.; Jiang, M.; Luo, Y.; Liao, W.; Wang, Y.; Gao, X.; Liu, J.; et al. The Rlm1 transcription factor in Candida oleophila contributes to abiotic stress resistance and biocontrol efficacy against postharvest gray mold of kiwifruit. Postharvest Biol. Technol. 2020, 166. [CrossRef] 